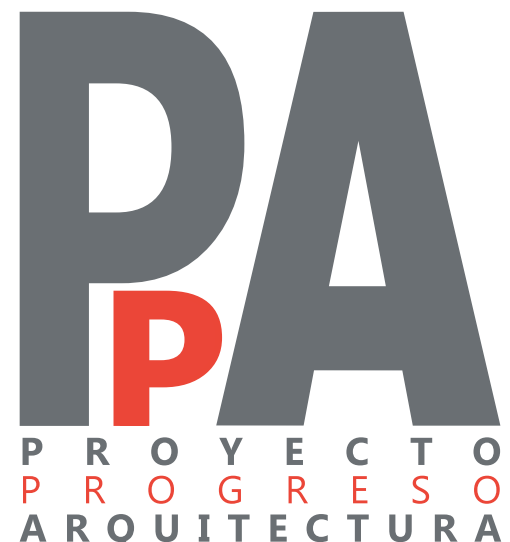

ARQUITECTURAS AMPLIADAS

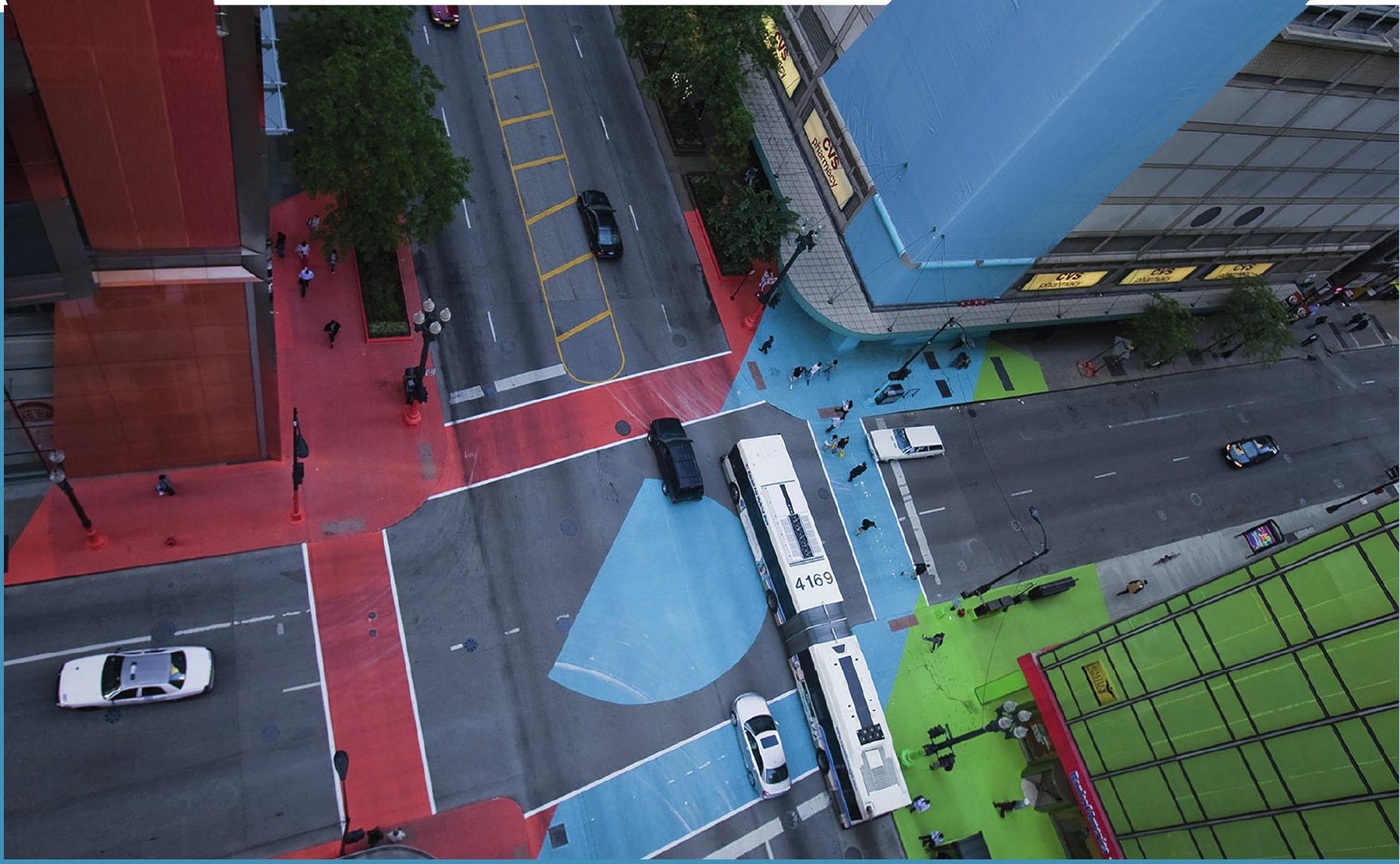




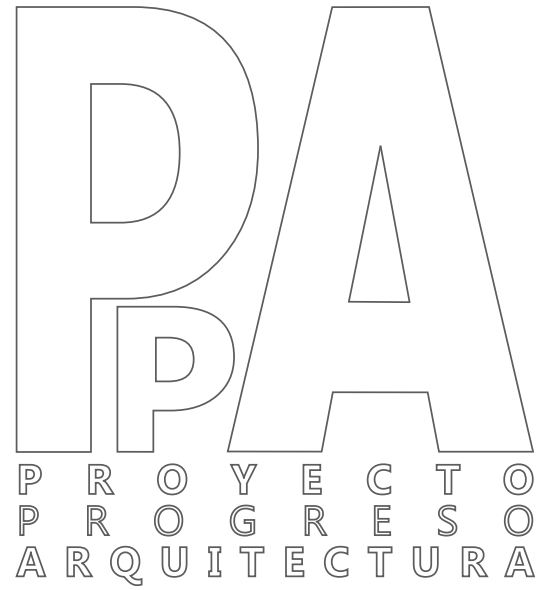

\section{ARQUITECTURAS AMPLIADA}

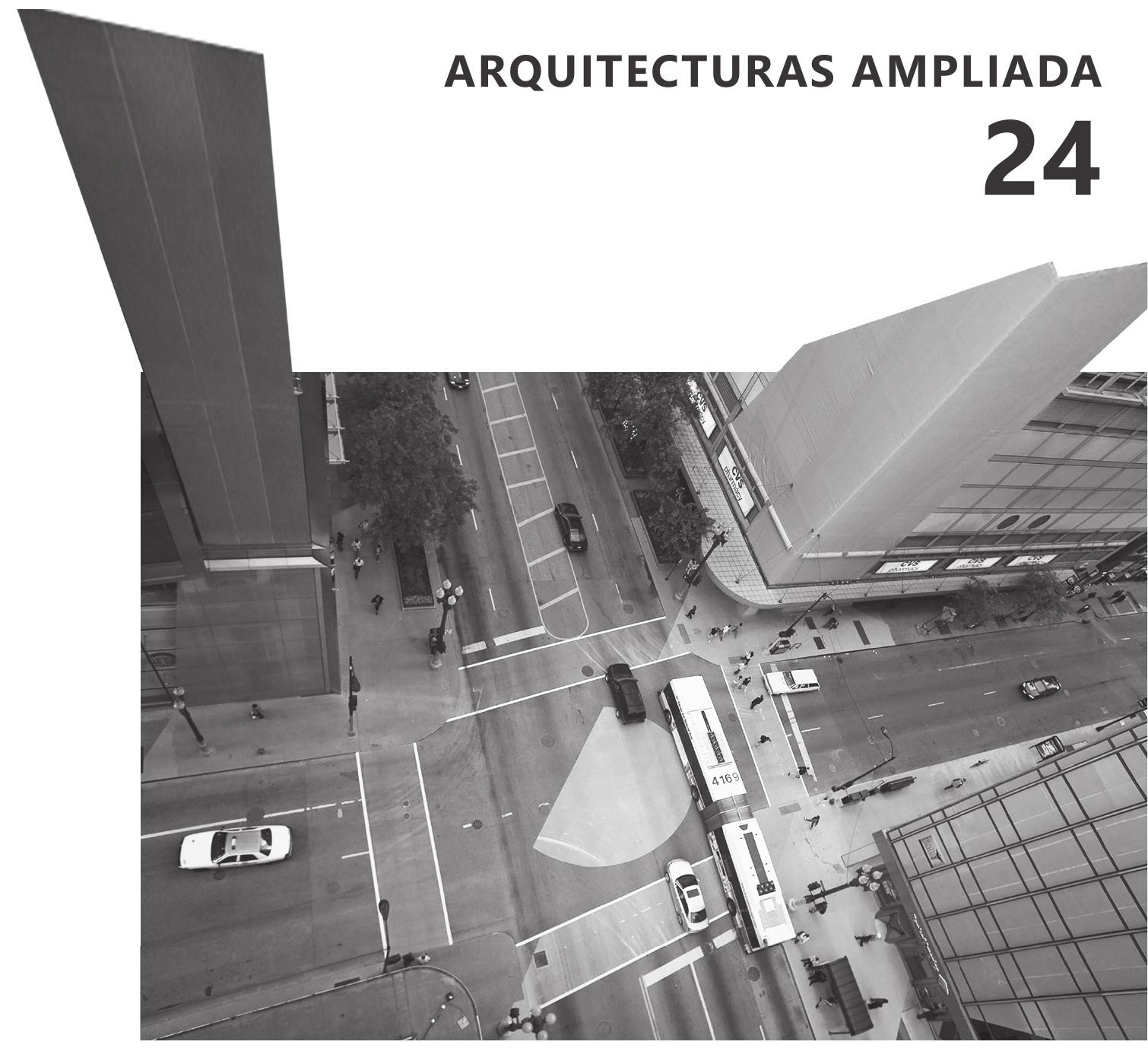

EDITORIAL UNIVERSIDAD DE SEVILLA AÑO 2019. ISSN 2171-6897 ISSNe 2173-1616 DOI: http://dx.doi.org/10.12795/ppa 


\section{REVISTA PROYECTO PROGRESO ARQUITECTURA}

N2/4

arquitecturas ampliadas
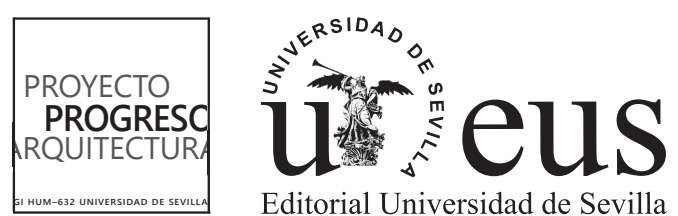


\section{arquitecturas ampliadas}

EDITA

Editorial Universidad de Sevilla. Sevilla

DIRECCIÓN CORRESPONDENCIA CIENTÍFICA

E.T.S. de Arquitectura. Avda Reina Mercedes, nº $241012-$

Sevilla.

Amadeo Ramos Carranza, Dpto. Proyectos Arquitectónicos.

e-mail: revistappa.direccion@gmail.com

\section{EDICIÓN ON-LINE}

Portal informático https://revistascientificas.us.es/index.php/ppa Portal informático Grupo de Investigación HUM-632

http://www. proyectoprogresoarquitectura.com

Portal informático Editorial Universidad de Sevilla http://www. editorial.us.es/

(c) EDITORIAL UNIVERSIDAD DE SEVILLA, 2019.

Calle Porvenir, 27. 41013 SEVILLA. Tfs. 954487447 / 954487451

Fax 954487443. [eus4@us.es] [http://www.editorial.us.es]

(c) TEXTOS: SUS AUTORES,

(C) IMÁGENES: SUS AUTORES Y/O INSTITUCIONES

DISEÑO PORTADA:

Rosa María Añón Abajas - Amadeo Ramos Carranza

En base a la fotografía: (C) Jessica Stockholder, cortesía de la artista y de Mitchell-Innes \& Nash, Nueva York

\section{DISEÑO PLANTILLA PORTADA-CONTRAPORTADA}

Miguel Ángel de la Cova Morillo-Velarde

DISEÑO PLANTILLA MAQUETACIÓN

Maripi Rodríguez

MAQUETACIÓN

Referencias Cruzadas

CORRECCION ORTOTIPOGRÁFICA

José Antonio Duarte

ISSN (ed. impresa): 2171-6897

ISSN-e (ed. electrónica): 2173-1616

DOI: http://dx.doi.org/10.12795/ppa

DEPÓSITO LEGAL: SE-2773-2010

PERIOCIDAD DE LA REVISTA: MAYO Y NOVIEMBRE

IMPRIME: PODIPRINT

Reservados todos los derechos. Ni la totalidad ni parte de esta revista puede reproducirse o transmitirse por ningún procedimiento electrónico o mecánico, incluyendo fotocopia, grabación magnética o cualquier almacenamiento de información y sistema de recuperación, sin permiso escrito de la Editorial Universidad de Sevilla.

Las opiniones y los criterios vertidos por los autores en los artículos firmados son responsabilidad exclusiva de los mismos. 


\section{DIRECCIÓN}

Dr. Amadeo Ramos Carranza. Escuela Técnica Superior de Arquitectura. Universidad de Sevilla. España

\section{SECRETARÍA}

Dra. Rosa María Añón Abajas. Escuela Técnica Superior de Arquitectura. Universidad de Sevilla. España

\section{EQUIPO EDITORIAL \\ Edición:}

Dr. Amadeo Ramos Carranza. Escuela Técnica Superior de Arquitectura. Universidad de Sevilla. España.

Dra. Rosa María Añón Abajas. Escuela Técnica Superior de Arquitectura. Universidad de Sevilla. España.

Dr. Francisco Javier Montero Fernández. Escuela Técnica Superior de Arquitectura. Universidad de Sevilla. España.

Dra. Esther Mayoral Campa. Escuela Técnica Superior de Arquitectura. Universidad de Sevilla. España.

Dr. Miguel Ángel de la Cova Morillo-Velarde. Escuela Técnica Superior de Arquitectura. Universidad de Sevilla. España.

Dr. Germán López Mena. Escuela Técnica Superior de Arquitectura. Universidad de Sevilla. España.

Dra. Gloria Rivero Lamela. Escuela Técnica Superior de Arquitectura. Universidad de Sevilla. España.

Juan José López de la Cruz. Escuela Técnica Superior de Arquitectura. Universidad de Sevilla. España.

Guillermo Pavón Torrejón. Escuela Técnica Superior de Arquitectura. Universidad de Sevilla. España.

Externos edición (asesores):

Dr. José Altés Bustelo. Escuela Técnica Superior de Arquitectura. Universidad de Valladolid. España.

Dr. Carlos Arturo Bell Lemus. Facultad de Arquitectura. Universidad del Atlántico. Colombia.

Dr. José de Coca Leicher. Escuela Técnica Superior de Arquitectura. Universidad Politécnica de Madrid. España. Dra. Patricia de Diego Ruiz. Escuela Técnica Supeiror de Arquitectura y Geodesia. Universidad Alcalá de Heranes. España.

Dr. Alfonso del Pozo y Barajas. Escuela Técnica Superior de Arquitectura. Universidad de Sevilla. España.

Dr. Jaume J. Ferrer Fores. Escola Tècnica Superior

d'Arquitectura de Barcelona. Universitat Politècnica de Catalunya. España.

Dra. Laura MArtínez Guereñu. El School of Architecture \& Design, IE University, Madrid; Segovia. España.

Dra. Clara Mejía Vallejo. Escuela Técnica Superior de Arquitectura. Universidad Politécnica de Valencia. España.

Dra. Luz Paz Agras. Escuela Técnica Superior de Arquitectura. Universidade da Coruña. España.

Dra. Marta Sequeira. CIAUD, Faculdade de Arquitectura da Universidade de Lisboa, Portugal.

\section{SECRETARÍA TÉCNICA}

Dra. Gloria Rivero Lamela. Escuela Técnica Superior de Arquitectura. Universidad de Sevilla. España.

\section{EDITORA Y COORDINACION CONTENIDOS CIENTÍFICOS DEL NÚMERO}

Dra. Rosa María Añón Abajas. Escuela Técnica Superior de Arquitectura. Universidad de Sevilla. España.

\section{COMITÉ CIÉNTIFICO}

Dr. Carlo Azteni. DICAAR. Dipartimento di Ingegneria Civile, Ambientale e Architettura. University Of Cagliari. Italia.

Dra. Maristella Casciato. GETTY Research Institute, GETTY, Los Angeles. Estados Unidos.

Dra. Anne Marie Châtelet. École Nationale Supérieure D'Architecture de Strasbourg (ENSAS). Francia.

Dr. Jean Louis Cohen. Institute of Fine Arts, New York University. Estados Unidos.

Dra. Josefina González Cubero. Escuela Técnica Superior de Arquitectura. Universidad de Valladolid. España.

Dr. José Manuel López Peláez. Escuela Técnica Superior de Arquitectura. Universidad Politécnica de Madrid. España.

Dra. Maite Méndez Baiges. Departamento de Historia del Arte. Universidad de Málaga. España.

Dr. Dietrich C. Neumann. Brown University In Providence, Ri (John Nicholas Brown Center For Public Humanities And Cultural Heritage). Estados Unidos.

Dr. Víctor Pérez Escolano. Catedrático Historia, Teoría y Composición Arquitectónicas. Escuela Técnica Superior de Arquitectura. Universidad de Sevilla. España.

Dr. Jorge Torres Cueco. Catedrático Proyectos Arquitectónicos. Escuela Técnica Superior de Arquitectura. Universitat Politècnica de València. España.

Dr. ir. Frank van der Hoeven, TU DELFT. Architecture and the Built Environment, Netherlands

\section{CORRESPONSALES}

Pablo de Sola Montiel. The Berlage Centre for Advanced Studies in Architecture and Urban Design. Paises Bajos.

Dr. Plácido González Martínez. Tongji University Caup (College Of architectura \& Urban Planing). Shangai, China.

Patrícia Marins Farias. Faculdade de Arquitetura. Universidade Federal da Bahia. Brasil.

Dr. Daniel Movilla Vega. Umeå School of Architecture. Umeå University. Suecia.

Dr. Pablo Sendra Fernández. The Bartlett School of Planning. University College London. Inglaterra.

Alba Zarza Arribas. Centro de Estudos Arnaldo Araújo, Porto. Portugal.

Dra. María Elena Torres Pérez. Facultad de Arquitectura. Universidad Autónoma de Yucatán, Mérida. México.

\section{TEXTOS VIVOS}

Juan José López de la Cruz. Escuela Técnica Superior de Arquitectura. Universidad de Sevilla. España.

Dr. Francisco Javier Montero Fernández. Escuela Técnica Superior de Arquitectura. Universidad de Sevilla. España. Dra. Esther Mayoral Campa. Escuela Técnica Superior de Arquitectura. Universidad de Sevilla. España. 


\section{SERVICIOS DE INFORMACIÓN}

\section{CALIDAD EDITORIAL}

La Editorial Universidad de Sevilla cumple los criterios establecidos por la Comisión Nacional Evaluadora de la Actividad Investigadora para que lo publicado por el mismo sea reconocido como "de impacto" (Ministerio de Ciencia e Innovación, Resolución 18939 de 11 de noviembre de 2008 de la Presidencia de la CNEAI, Apéndice I, BOE n² 282, de 22.11.08).

La Editorial Universidad de Sevilla forma parte de la U.N.E. (Unión de Editoriales Universitarias Españolas) ajustándose al sistema de control de calidad que garantiza el prestigio e internacionalidad de sus publicaciones.

PUBLICATION QUALITY

The Editorial Universidad de Sevilla fulfils the criteria established by the National Commission for the Evaluation of Research Activity (CNEAI) so that its publications are recognised as "of impact" (Ministry of Science and Innovation, Resolution 18939 of 11 November 2008 on the Presidency of the CNEAl, Appendix I, BOE No 282, of 22.11.08).

The Editorial Universidad de Sevilla operates a quality control system which ensures the prestige and international nature of its publications, and is a member of the U.N.E. (Unión de Editoriales Universitarias Españolas-Union of Spanish University Publishers).

Los contenidos de la revista PROYECTO, PROGRESO, ARQUITECTURA aparecen en:

bases de datos: indexación
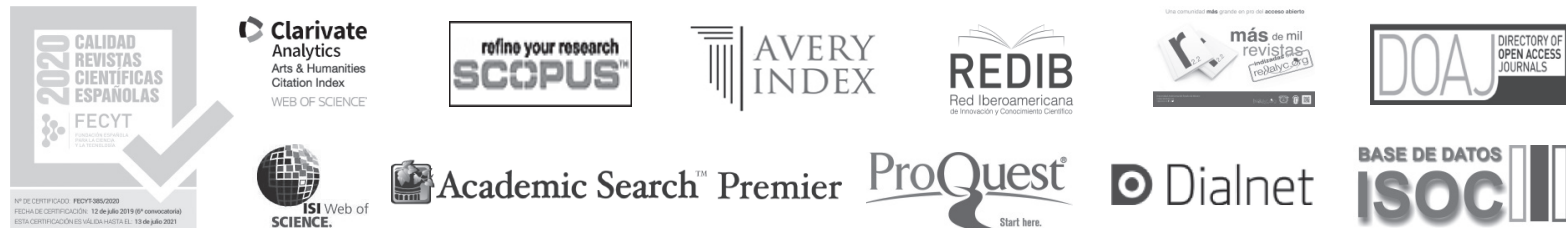

D Dialnet

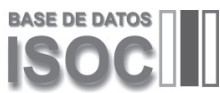

SELLO DE CALIDAD EDITORIAL FECYT 2019. RENOVADO 2020. (Cuartil C3)

WoS. Arts \& Humanities Citation Index

WoS. ESCl - Emerging Sources Citation Index

SCOPUS

AVERY. Avery Index to Architectural Periodicals

REBID. Red Iberoamericana de Innovación y Conocimiento Científico

REDALYC. Red de Revistas Científicas de América Latina y el Caribe, España y Portugal.

EBSCO. Fuente Académica Premier

EBSCO. Art Source

DOAJ, Directory of Open Access Journals

PROQUEST (Arts \& Humanities, full text)

DIALNET

ISOC (Producida por el CCHS del CSIC)

DRIJ. Directory of Research Journals Indexing

SJR (2019): 0.100, H index: 2

\section{catalogaciones: criterios de calidad}

RESH (Revistas Españolas de Ciencias Sociales y Humanidades).

Catálogos CNEAI (16 criterios de 19). ANECA (18 criterios de 21). LATINDEX (35 criterios sobre 36).

DICE (CCHS del CSIC, ANECA).

MIAR, Matriu d'Informació per a l'Avaluació de Revistes. IDCS 2018: 10,500. Campo ARQUITECTURA

CLASIFICACIÓN INTEGRADA DE REVISTAS CIENTÍFICAS (CIRC-CSIC): A

ERIHPLUS

SCIRUS, for Scientific Information.

ULRICH'S WEB, Global Serials Directory.

ACTUALIDAD IBEROAMERICANA.

\section{catálogos on-line bibliotecas notables de arquitectura:}

CLIO. Catálogo on-line. Columbia University. New York

HOLLIS. Catálogo on-line. Harvard University. Cambridge. MA

SBD. Sistema Bibliotecario e Documentale. Instituto Universitario di Architettura di Venezia

OPAC. Servizi Bibliotecari di Ateneo. Biblioteca Centrale. Politecnico di Milano

COPAC. Catálogo colectivo (Reino Unido)

SUDOC. Catálogo colectivo (Francia)

ZBD. Catálogo colectivo (Alemania)

REBIUN. Catálogo colectivo (España)

OCLC. WorldCat (Mundial) 
EVALUACIÓN EXTERNA POR PARES Y ANÓNIMA.

El Consejo Editorial remitirá el artículo a dos expertos revisores anónimos dentro del campo específico de investigación y crítica de arquitectura, según el modelo doble ciego.

El director de la revista comunicará a los autores el resultado motivado de la evaluación por correo electrónico, en la dirección que éstos hayan utilizado para enviar el artículo. El director comunicará al autor principal el resultado de la revisión (publicación sin cambios; publicación con correcciones menores; publicación con correcciones importantes; no aconsejable para su publicación), así como las observaciones y comentarios de los revisores.

Si el manuscrito ha sido aceptado con modificaciones, los autores deberán reenviar una nueva versión del artículo, atendiendo a las demandas y sugerencias de los evaluadores externos. Los artículos con correcciones importantes serán remitidos al Consejo Asesor para verificar la validez de las modificaciones efectuadas por el autor. Los autores pueden aportar también una carta al Consejo Editorial en la que indicarán el contenido de las modificaciones del artículo. Los artículos con correcciones importantes serán remitidos al Consejo Asesor para verificar la validez de las modificaciones efectuadas por el autor.

\section{DECLARACIÓN ÉTICA SOBRE PUBLICACIÓN Y MALAS PRÁCTICAS}

La revista PROYECTO, PROGRESO, ARQUITECTURA (PPA) está comprometida con la comunidad académica en garantizar la ética y calidad de los artículos publicados. Nuestra revista tiene como referencia el Código de Conducta y Buenas Prácticas que, para editores de revistas científicas, define el COMITÉ DE ÉTICA DE PUBLICACIONES (COPE).

Así nuestra revista garantiza la adecuada respuesta a las necesidades de los lectores y autores, asegurando la calidad de lo publicado, protegiendo y respetando el contenido de los artículos y la integridad de los mismo. El Consejo Editorial se compromete a publicar las correcciones, aclaraciones, retracciones y disculpas cuando sea preciso.

En cumplimiento de estas buenas prácticas, la revista PPA tiene publicado el sistema de arbitraje que sigue para la selección de artículos así como los criterios de evaluación que deben aplicar los evaluadores externos -anónimos y por pares, ajenos al Consejo Editorial-. La revista PPA mantiene actualizados estos criterios, basados exclusivamente en la relevancia científica del artículo, originalidad, claridad y pertinencia del trabajo presentado.

Nuestra revista garantiza en todo momento la condifencialidad del proceso de evaluación: el anonimato de los evaluadores y de los autores; el contenido evaluado; los informes razonados emitidos por los evaluadores y cualquier otra comunicación emitida por los consejos Editorial, Asesor y Científico si así procediese.

Igualmente quedan afectados de la máxima confidencialidad las posibles aclaraciones, reclamaciones o quejas que un autor desee remitir a los comités de la revista o a los evaluadores del artículo.

La revista PROYECTO, PROGRESO, ARQUITECTURA (PPA) declara su compromiso por el respeto e integridad de los trabajos ya publicados. Por esta razón, el plagio está estrictamente prohibido y los textos que se identifiquen como plagio o su contenido sea fraudulento, serán eliminados o no publicados por la revista PPA. La revista actuará en estos casos con la mayor celeridad posible. Al aceptar los términos y acuerdos expresados por nuestra revista, los autores han de garantizar que el artículo y los materiales asociados a él son originales o no infringen derechos de autor. También los autores tienen que justificar que, en caso de una autoría compartida, hubo un consenso pleno de todos los autores afectados y que no ha sido presentado ni publicado con anterioridad en otro medio de difusión.

\section{EXTERNAL ANONYMOUS PEER REVIEW.}

Editorial Board will be sent to two anonymous experts, within the specific field of architectural investigation and critique, for a double blind review.

The Director of the journal will communicate the result of the reviewers evaluations to the authors by electronic mail, to the address used to send the article. The Director will communicate the result of the review (publication without changes; publication with minor corrections; publication with significant corrections; its publication is not advisable), as well as the observations and comments of the reviewers, to the main author.

If the manuscript has been accepted with modifications, the authors will have to resubmit a new version of the article, addressing the requirements and suggestions of the external reviewers. The articles with corrections will be sent to Advisory Board for verification of the validity of the modifications made by the author. The authors can also send a letter to the Editorial Board, in which they will indicate the content of the modifications of the article.

\section{ETHICS STATEMENT ON PUBLICATION AND BAD PRACTICES} PROYECTO, PROGRESO ARQUITECTURA (PPA) makes a commitment to the academic community by ensuring the ethics and quality of its published articles. As a benchmark, our journal uses the Code of Conduct and Good Practices which, for scientific journals, is defined for editors by the PUBLICATION ETHICS COMMITTEE (COPE).

Our journal thereby guarantees an appropriate response to the needs of readers and authors, ensuring the quality of the published work, protecting and respecting the content and integrity of the articles. The Editorial Board will publish corrections, clarifications, retractions and apologies when necessary.

In compliance with these best practices, PPA has published the arbitration system that is followed for the selection of articles as well as the evaluation criteria to be applied by the anonymous, external peer-reviewers. PPA keeps these criteria current, based solely on the scientific importance, the originality, clarity and relevance of the presented article.

Our journal guarantees the confidentiality of the evaluation process at all times: the anonymity of the reviewers and authors; the reviewed content; the reasoned report issued by the reviewers and any other communication issued by the editorial, advisory and scientific boards as required.

Equally, the strictest confidentiality applies to possible clarifications, claims or complaints that an author may wish to refer to the journal's committees or the article reviewers.

PROYECTO, PROGRESO ARQUITECTURA (PPA) declares its commitment to the respect and integrity of work already published. For this reason, plagiarism is strictly prohibited and texts that are identified as being plagiarized, or having fraudulent content, will be eliminated or not published in PPA. The journal will act as quickly as possible in such cases. In accepting the terms and conditions expressed by our journal, authors must guarantee that the article and the materials associated with it are original and do not infringe copyright. The authors will also have to warrant that, in the case of joint authorship, there has been full consensus of all authors concerned and that the article has not been submitted to, or previously published in, any other media. 
EVALUADORES EXTERNOS (publicación cada cuatro números, dos años). NÚMEROS 21 a 24 (incluidos)

Álvarez Álvarez, Darío. Catedrático de Universidad / Departamento de Teoría de la Arquitectura y Proyectos Arquitectónicos / ETS Arquitectura / Universidad de Valladolid / España.

Arrieta Berdasco, Valentín. Doctor Arquitecto, Profesor Asociado / Departamento de Teoría de la Arquitectura y Proyectos Arquitectónicos / ETS Arquitectura / Universidad de Valladolid / España.

Bardí i Milá, Berta. Profesora Asociada doctora / / Departamento de Projectes Arquitectònics / ETS Arquitectura / Universitat Politècnica de Catalunya, Barcelona Tech / España.

Bergera Serrano, Iñaki. Titular de Universidad / Unidad Predepartamental de Arquitectura / Área Proyectos Arquitectónicos / El y Arquitectura / Universidad de Zaragoza / España.

Bobbink, Inge. Dr. ir. Architecture / Section of landscape architecture / TU Delft / Países Bajos.

Burriel Bielza, Luis. Profesor Titular / École Nationale Supérieure d'Architecture de Paris-Belleville / Francia

Calatrava Escobar, Juan. Catedrático de Universidad / Departamento de Construcciones Arquitectónicas / ETS Arquitectura / Universidad de Granada / España.

Castellanos Gómez, Raúl. Titular de Universidad / Departamento de Proyectos Arquitectónicos / ETS Arquitectura / Universidad Politécnica de Valencia / España.

Centellas Soler, Miguel. Titular de Universidad / Departamento de Arquitectura y Tecnología de la Edificación / ETS Arquitectura y Edificación / Universidad Politécnica de Cartagena / España.

Chías Navarro, Pilar. Catedrática de Universidad / Departamento de Arquitectura / ETS Arquitectura y Geodesia / Universidad de Alcalá de Henares / España.

de Diego Ruiz, Patricia. Doctora arquitecta, Profesora Asociada / Departamento de Arquitectura / ETS Arquitectura y Geodesia / Universidad de Alcalá de Henares / España.

de la Iglesia Salgado, Félix. Profesor Contratado Doctor / Departamento de Proyectos Arquitectónicos / ETS Arquitectura / Universidad de Sevilla / España.

de la O Cabrera, Manuel Rodrigo. Doctor Arquitecto, Profesor Asociado / Departamento de Composición Arquitectónica / ETS Arquitectura / Universidad Politécnica de Madrid / España.

Delgado Orusco, Eduardo. Profesor Ayudante Doctor / Departamento de Arquitectura. Área de Proyectos Arquitectónicos / Escuela de Ingeniería y Arquitectura / Universidad de Zaragoza / España.

Deltell Pastor, Juan. Titular de Universidad / Departamento de Proyectos Arquitectónicos / ETS Arquitectura / Universitat Politècnica de València / España.

Diañez Rubio, Pablo. Titular de Universidad / Departamento de Proyectos Arquitectónicos / ETS Arquitectura / Universidad de Sevilla / España.

Díaz Segura, Alfonso. Titular de Universidad / Proyectos, Teoría y Técnica del Diseño y la Arquitectura / ETS Arquitectura / Universidad CEU Cardenal Herrera, Valencia / España.

Domingo Calabuig, Débora. Titular de Universidad / Departamento de Proyectos Arquitectónicos / ETS Arquitectura / Universidad Politécnica de Valencia / España.

Fernández Fariña, Almudena. Profesora Contratada Doctora / Departamento de Pintura / Facultad de Bellas Artes / Universidad de Vigo / España.

Fernández-Trapa de Isasi, Justo. Catedrático de Universidad / Departamento de Proyectos Arquitectónicos / ETS Arquitectura / Universidad Politécnica de Madrid / España.

García Escudero, Daniel. Dr. Arquitecto, Profesor Lector / Departamento de Projectes Arquitectònics / ETS Arquitectura / Universitat Politècnica de Catalunya, Barcelona Tech / España.

González Cubero, Josefina. Titular de Universidad / Departamento de Teoría de la Arquitectura y Proyectos Arquitectónicos / ETS Arquitectura / Universidad de Valladolid / España.

González Fraile, Eduardo. Catedrático de Universidad / Departamento de Teoría de la Arquitectura y Proyectos Arquitectónicos / ETS Arquitectura / Universidad de Valladolid / España.

Gorostiza López, Jorge. Doctor Arquitecto. Cineasta / España.

Hernández Moreno, Silverio. Profesor investigador titular / Facultad de Arquitectura y Diseño / Universidad Autónoma del Estado de México / México.

Labarta Aizpún, Carlos. Titular de Universidad / Unidad Predepartamental de Arquitectura / Área Proyectos Arquitectónicos / Escuela de Ingeniería y Arquitectura / Universidad de Zaragoza / España.

Lizondo Sevilla, Laura. Titular de Universidad / Departamento de Proyectos Arquitectónicos / ETS Arquitectura / Universidad Politécnica de Valencia / España.

Llopis Verdú, Jorge. Catedrático de Universidad / Departamento de Expresión Gráfica Arquitectónica / ETS Arquitectura / Universidad Politécnica de Valencia / España.

López Bahut, Emma. Profesora Contratada Doctora / Departamento de Proyectos arquitectónicos, Urbanismo y Composición / ETS Arquitectura / Universidade da Coruña / España.

López Fernández, Andrés. Titular de Universidad / Departamento de Proyectos Arquitectónicos / ETS Arquitectura / Universidad de Sevilla / España.

Loren Méndez, Mar. Catedrática de Universidad / Departamento de Historia, Teoría y Composición Arquitectónica / ETS Arquitectura / Universidad de Sevilla / España.

Maino Ansaldo, Sandro. Doctor Arquitecto / Departamento Arquitectura / Universidad Técnica Federico Santa María / Chile.

Mària i Serrano, Magda. Profesora Contratada Doctor / Departamento de Projectes Arquitectònics / ETS Arquitectura del Vallès / Universitat Politècnica de Catalunya / España

Marson, Anna. Profesora Ordinario / Dipartimento di culture del progetto / Istituto Universitario di Architettura di Venezia / Università di Venezia / Italia.

Martínez Díaz, Ángel. Titular de Universidad / Departamento de Ideación Gráfica Arquitectónica / ETS Arquitectura / Universidad Politécnica de Madrid / España. 
Mejía Vallejo, Clara. Titular de Universidad / Departamento de Proyectos Arquitectónicos / ETS Arquitectura / Universidad Politécnica de Valencia / España.

Mercader Moyano, Pilar. Titular de Universidad / Departamento de Proyectos Arquitectónicos / ETS Arquitectura / Universidad de Sevilla / España.

Mercé Hospital, José María. Catedrático de Universidad / Departamento de Proyectos Arquitectónicos / ETS Arquitectura y Geodesia / Universidad Alcalá de Henares / España.

Merí de la Maza, Ricardo. Titular de Universidad / Departamento de Proyectos Arquitectónicos / ETS Arquitectura / Universidad Politécnica de Valencia / España.

Mestre Martínez, Nieves. Doctora arquitecta, Profesora Asociada / Departamento de Proyectos Arquitectónicos / ETS Arquitectura / Universidad Politécnica de Madrid / España.

Millán Gómez, Antonio. Catedrático de Universidad / Departamento d’Expressió Gràfica Arquitectònica I / ETS Arquitectura del Vallès / Universitat Politècnica de Catalunya / España.

Moreno Pérez, José Ramón. Titular de Universidad / Dpto. Historia, Teoría y Composición Arquitectónica / ETS Arquitectura / Universidad de Sevilla / España.

Nijhuis, Steffen. Associate Professor. Head of Landscape Architecture Research / Section of Landscape Architecture / Department of Urbanism / Faculty of Architecture and the Built Environment / TU Delft / Países Bajos.

Ojeda Rivera, Juan Francisco. Catedrático de Universidad / Departamento de Geografía, Historia y Filosofía / Universidad Pablo de Olavide, Sevilla / España.

Paz-Agras, Luz. Profesora Ayudante doctor / Departamento de Proyectos Arquitectónicos, Urbanismo y Composición. Área de Composición Arquitectónica / ETS Arquitectura / Universidad de A Coruña / España

Pérez Moreno, Lucía C. Titular de Universidad / Departamento de Arquitectura. Área de Composición Arquitectónica / Escuela de Ingeniería y Arquitectura / Universidad de Zaragoza / España.

Pina Lupiañez, Rafael. Profesor Contratado Doctor / Departamento de Proyectos Arquitectónicos / ETS Arquitectura / Universidad Politécnica de Madrid / España

Oliverira do Nascimento, Francisco. Professor Auxiliar / Faculdade de Arquitetura / Universidade de Lisboa / Portugal.

Rovira Llobera, Teresa. Titular de Universidad / Departamento de Projectes Arquitectònics / ETS Arquitectura / Universitat Politècnica de Catalunya, Barcelona Tech / España.

Ruiz Rosa, José Antonio. Catedrático de Universidad / Departamento de Expresión Gráfica Arquitectónica / ETS Arquitectura / Universidad de Sevilla. / España.

Sabaté Bel, Joaquín. Catedrático de Universidad / Departamento de Urbanismo y Ordenación del Territorio / ETS Arquitectura / Universitat Politècnica de Catalunya, Barcelona Tech / España

Sainz Gutiérrez Victoriano. Titular de Universidad / Departamento de Urbanismo y Ordenación del Territorio / ETS Arquitectura / Universidad de Sevilla / España

Sánchez Lampreave, Ricardo. Profesor Titular / Área de Composición Arquitectónica / Escuela de Ingeniería y Arquitectura / Universidad de Zaragoza / España.

Sambricio R. Echegaray, Carlos. Catedrático de Universidad / Departamento de Composición Arquitectónica / ETS Arquitectura / Universidad Politécnica de Madrid / España. / España.

Santamarina-Macho, Carlos. España. Doctor Arquitecto, Profesor Asociado / Departamento de Teoría de la Arquitectura y Proyectos Arquitectónicos / ETS Arquitectura / Universidad de Valladolid / España

Senra Fernández-Miranda, Ignacio. Doctor Arquitecto, Profesor Asociado / Departamento de Proyectos Arquitectónicos / ETS Arquitectura / Universidad Politécnica de Madrid / España.

Sentieri Omarrementeria, Carla. Titular de Universidad / Departamento de Proyectos Arquitectónicos / ETS Arquitectura / Universidad Politécnica de Valencia / España.

Sequeira Marta. Professora doctora / ISCTE - Instituto Universitário de Lisboa/ Universidade Autónoma de Lisboa / CIAUD - Faculdade de Arquitectura da Universidade de Lisboa / Portugal.

Sola Alonso, José Ramón. España. Profesor Contratado Doctor / Departamento de Teoría de la Arquitectura y Proyectos Arquitectónicos / ETS Arquitectura / Universidad de Valladolid / España.

Trillo Martínez, Valentín. Doctor arquitecto Profesor Asociado / Departamento de Proyectos Arquitectónicos / ETS Arquitectura / Universidad de Sevilla / España.

Villalobos Alonso, Daniel. Titular de Universidad / Departamento de Teoría de la Arquitectura y Proyectos Arquitectónicos / ETS Arquitectura / Universidad de Valladolid / España.

Verde Zein, Ruth. Doctora arquitecto e investigadora / Facultad de Arquitectura y Urbanismo / Universidad Presbiteriana Mackenzie. São Paulo / Brasil.

\section{ESTADÍSTICAS PUBLICACIÓN (publicación cada cuatro números, dos años). NÚMEROS 1 a 20 (incluidos)}

Total artículos recibidos: 546

Total artículos publicados: 199 (36,45\%)

Total artículos rechazados: $347(63,55 \%)$

Total artículos publicados de autores pertenecientes a los diferentes consejos o comités organizadores de la revista y Grupo de Investigación "proyecto, progreso, arquitectura"(endogamia): 20 (10,05\%)

Total artículos publicados de autores pertenecientes a la Universidad de Sevilla: 53 (26,63\%)

Total artículos publicados de autores externos a los diferentes consejos o comités organizadores de la revista y Grupo de Investigación

"proyecto, progreso, arquitectura": 179 (89,95\%)

Total artículos publicados de autores extranjeros: 17 (8,54\%) 


\section{arquitecturas ampliadas}

índice

editorial

DESPEJAR LA ARQUITECTURA, LIBERAR EL ESPACIO Y AMPLIAR CONCEPTOS / UNCLUTTER ARCHITECTURE, FREE UP SPACE AND EXPAND CONCEPTS

Rosa María Añón-Abajas - (DOI: http://dx.doi.org/10.12795/ppa.2021.i24.10)

entre líneas

PAISAJES ARQUITECTÓNICOS Y MEMORIAS DE LA CIUDAD / ARCHITECTURAL LANDSCAPES AND MEMORIES OF THE CITY

Darío Álvarez Álvarez - (D0l: http://dx.doi.org/10.12795/ppa.2021.i24.01)

artículos

LINAZASORO EN REIMS. EL ESPACIO PÚBLICO COMO MEMORIA DEL LUGAR / LINAZASORO IN REIMS. THE PUBLIC SPACE AS THE MEMORY OF THE PLACE

Victoriano Sainz Gutiérrez - (DOl: http://dx.doi.org/10.12795/ppa.2021.i24.02)

EDITAR VS. CONSTRUIR: UNA ECOLOGÍA DE LO INVISIBLE. AMPLIFICAR LA COMPRENSIÓN DE LAS TÉCNICAS DE PROYECTO / EDITING VS. BUILDING: AN ECOLOGY OF THE INVISIBLE. AMPLIFYING THE UNDERSTANDING OF ARCHITECTURAL DESIGN TECHNIQUES

Paula Victoria Álvarez Benítez - (DOI: http://dx.doi.org/10.12795/ppa.2021.i24.03)

CUANDO LA PINTURA AMPLÍA LA ARQUITECTURA: INTERVENCIONES REALIZADAS EN EL ESPACIO PÚBLICO / WHEN PAINTING ENHANCES ARCHITECTURE: INTERVENTIONS IN A PUBLIC SETTING Aurora Alcaide-Ramírez; Ana Ruiz-Abellón - (D0l: http://dx.doi.org/10.12795/ppa.2021.i24.04)

UN EDIFICIO INVISIBLE. NUEVO AULARIO DE LA FACULTAD DE DERECHO DE LA UNIVERSIDAD DE ZARAGOZA (1983-1996) / AN INVISIBLE BUILDING. NEW LECTURE ROOM BUILDING OF THE FACULTY OF LAW OF THE UNIVERSITY OF ZARAGOZA (1983-1996)

Luis Miguel Lus-Arana; Lucía Carmen Pérez-Moreno - (D0l: http://dx.doi.org/10.12795/ppa.2021.i24.05)

ARQUITECTURAS AMPLIADAS. EL PABELLÓN DE EXPOSICIONES EN LA CASA DE CAMPO DE MADRID) / EXPANDED ARCHITECTURES. THE EXHIBITION PAVILION AT THE CASA DE CAMPO IN MADRID José de Coca Leicher - (DOI: http://dx.doi.org/10.12795/ppa.2021.i24.06)

reseña bibliográfica TEXTOS VIVOS

RAFAEL MONEO VALLÉS: LA VIDA DE LOS EDIFICIOS. LA MEZQUITA DE CÓRDOBA, LA LONJA DE SEVILLA Y UN CARMEN EN GRANADA

Víctor Pérez Escolano - (DOl: http://dx.doi.org/10.12795/ppa.2021.i24.07)

FRANCISCO DE GRACIA: CONSTRUIR EN LO CONSTRUIDO. LA ARQUITECTURA COMO MODIFICACIÓN

Pablo Diañez Rubio - (DOl: http://dx.doi.org/10.12795/ppa.2020.i24.08)

FRÉDÉRIC DRUOT, ANNE LACATON \& JEAN-PHILIPPE VASSAL PLUS: LA VIVIENDA COLECTIVA. TERRITORIO DE EXCEPCIÓN

Javier Terrados Cepeda - (DOI: http://dx.doi.org/10.12795/ppa.2021.i24.09) 

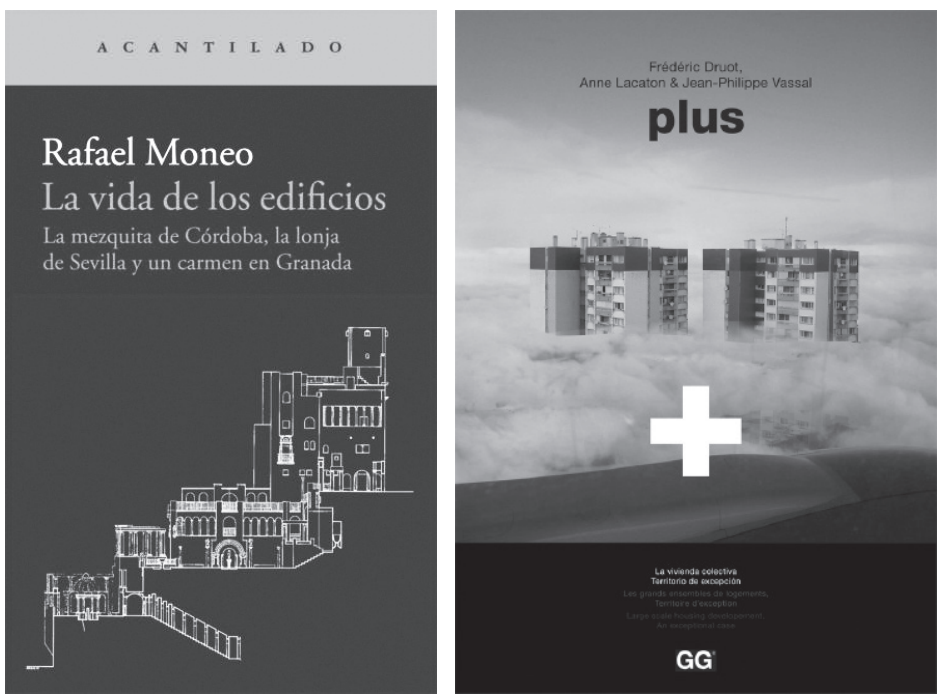

FRANCISCO DE GRACIA
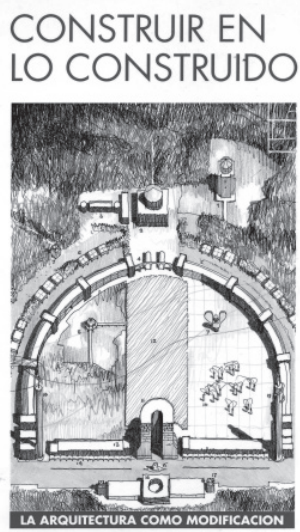

NEREA

\section{reseña bibliográfica TEXTOS VIVOS}

Nuestra época está sometida a transformaciones hasta ahora insospechadas a cuya aparición no somos ajenos y que afectan a la forma de entender y practicar la arquitectura. El entendimiento y la acción en la nueva arquitectura no deben abordarse solo desde la racionalidad del proyecto sino desde la reconstrucción crítica de la memoria de nuestra cultura y de nuestra participación en ella a lo largo del tiempo y en la evolución de la sociedad.

Cada tiempo, y el nuestro también, decide qué arquitectos y cuáles textos y obras han de ser rescatados y recalificados como clásicos.

Mediante el diálogo con ellos, los arquitectos actuales nos alinearemos en la tradición arquitectónica de la que, hoy, de manera perentoria, no es posible ni razonable prescindir. PROYECTO, PROGRESO, ARQUITECTURA destina esta sección a realizar un repaso propositivo y abierto a esos textos. 


\title{
FRANCISCO DE GRACIA: CONSTRUIR EN LO CONSTRUIDO. LA ARQUITECTURA COMO MODIFICACIÓN
}

\author{
Hondarribia (Guipúzcoa): Editorial Nerea, 1992, 3. ${ }^{a}$ edición, 2001, 324 páginas, formato 18 × 24 cm. ISBN: 84-86763-65-7.
}

Pablo Diañez Rubio (https://orcid.org/0000-0001-6338-5858)

Doctor arquitecto. Titular de universidad. Departamento de Proyectos Arquitectónicos. Escuela Técnica Superior de Arquitectura. Universidad de Sevilla. España.

Persona de contacto: pdianez@us.es

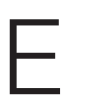

I objetivo de estas notas es intentar trasladar a un joven lector que aún no alcanzó la treintena al contexto que dio lugar al ensayo de Francisco de Gracia, cuya redacción se sitúa en 1991, aunque la edición que se comenta fue revisada en 2001. Para ello, deberá situarse en los años cincuenta del siglo pasado, en los que coincidieron varias líneas de investigación sobre el hecho arquitectónico.

En ellas se trató de hacer verificable el proceso de generación de la forma en arquitectura (C. Alexander, 1966), se apostó por considerar la ciudad como arquitectura (A. Rossi, 1966) y se hizo una seria crítica a algunos principios del movimiento moderno (R. Venturi, 1966). Una línea diferente fueron las llamadas metodologías del diseño que, iniciadas en el Congreso de Portsmouth (1958), culminan en el de Castelldefels (1972). La cuestión urbana también fue abordada desde una perspectiva innovadora (M. Castells, 1974) y el concurso de San Francisco el Grande ganado por J. Navarro Baldeweg (Madrid, 1982) marcó un hito sobre como renovar la ciudad heredada.

El texto que comento es deudor de aquella inquietud que trataba de establecer certezas sobre cómo se debía proyectar, sobre cuáles eran los criterios oportunos para abordar la producción formal y también sobre cómo se insertaba la nueva arquitectura en los centros históricos.

La obra se inicia con dos capítulos, "La ciudad como arquitectura" y "Modernidad y contexto", ambos de carácter teórico, en los que se presentan las circunstancias históricas que concurrieron para que a partir de 1918 se iniciase un proceso de ruptura con la producción de la obra artística y en particular con la nueva ciudad. A través de ejemplos principalmente del periodo aludido, desarrolla las claves que, a su juicio, fueron determinantes: abstracción contra figuración, la cuestión tipológica y el orden de la forma construida. Aloïs Riegl, Quatremère de Quincy y Rudolf Arnheim, entre otros, sirven de referentes para sustentar la insuficiencia del movimiento moderno respecto a los valores tradicionales de la ciudad y la arquitectura.

En la tercera parte del ensayo (La acción modificadora), desarrolla de forma didáctica la opción que sostiene el autor ante la situación descrita en las dos anteriores. A través de un estudio de casos que abarcan desde los primeros años cincuenta hasta finales de los ochenta, se redacta el capítulo sustantivo del trabajo y que da nombre al ensayo, "Construir en lo construido". En él desarrolla las líneas de análisis que le permiten pormenorizar sus posiciones y que atienden respectivamente al grado de intervención, a los criterios utilizados en cada caso y a las actitudes frente al contexto. Y aclara que... "nuestra pretensión consiste en reconocer distintos modos de intervención a través de una serie de ejemplos recogidos sin ánimo de ser exhaustivos, aunque en un número suficiente como para advertir la existencia de una panoplia de fórmulas cuyo trasfondo, y este sería un argumento básico de nuestro trabajo, nos permitiría hablar de una teoría general de la modificación, que no debe confundirse con una doctrina o una tratadística" (p. 186).

Diferencia tres variantes en función de que se trate de una modificación circunscrita al edificio como realidad individual, como es el caso, entre otros ejemplos, de la obra de Carlo Scarpa en Castelvecchio (Verona, 1964); que se trate de una modificación del locus, como se hace en la sede de The Economist (Londres, 1967), obra de Alison y Peter Smithson, en la que "los círculos progresistas de entonces facilitaron la ejecución de unos ortoprismas exentos capaces de alterar radicalmente la impronta del lugar" (p. 217). 
Por último, se alude a la renovación urbana de amplios fragmentos de tejido, como fue el caso de la IBA de Berlín (1984), la remodelación de Les Halles, en París (1976) o la reordenación del entorno de San Francisco el Grande, en Madrid (1982).

El apartado dedicado a los patrones de actuación aborda las técnicas de contextualización, como las empleadas en la conformación del tejido urbano del proyecto para el Derby Town Center (1970) de J. Stirling y L. Krier o el edificio de viviendas de Ignacio Gardella en el canal de La Giudecca (Venecia, 1958), al que califica como continuidad de imagen en línea con el edificio de La Previsión Española, de R. Moneo, en Sevilla (1987).

La recreación de formas tipológicas y la colisión de estructuras formales completan este apartado. Los edificios citados para este último patrón son el Museo Guggenheim (F. L. Wright, Nueva York, 1958), el Centro Pompidou (R. Piano, R. Rogers, París, 1977) y la Casa del Fascio (G. Terragni, Como, 1936). En todos ellos se pone en evidencia la autonomía de las opciones, con independencia de otras consideraciones.

La acción modificadora es considerada en un tercer y último apartado bajo la perspectiva de las actitudes frente al contexto. En esta ocasión, se parte de considerar tres diferentes posiciones ante el problema de la integración de la arquitectura moderna en los centros históricos: confirmar la confrontación mediante la descontextualización, promover una arquitectura abiertamente historicista o propiciar "una arquitectura que con un plus intencional de diseño, llegara a superar la supuesta imposibilidad original para integrarse en los centros históricos sin renunciar a su condición de moderna" (p. 288). En esta ocasión se identifican actitudes muy diversas con sus correspondientes ejemplos ilustrativos, como es el caso del Colegio de Arquitectos de Sevilla (E. Perea y G. Ruiz Cabrero, 1982) o la Banca Popular de Verona (C. Scarpa, 1981), a las que define como una arquitectura contextual.

La reciente muerte del filántropo estadounidense Richard Driehaus coincide con la reapertura de un debate que configura el argumento principal de la obra que se comenta, tradición y modernidad: "El financiero creó el premio de arquitectura tradicional que lleva su nombre y el galardón Rafael Manzano a la nueva arquitectura tradicional" (A. Zabalbeascoa, El País, 15 de marzo de 2021). Se trata de una opción cultural recurrente, y no solo en el ámbito de la arquitectura, pues Mon oncle (Jacques Tati, 1958), entre otras películas, trataron con notable acierto cómo era el tránsito de una a otra.

Bien ilustrado y con una redacción muy cuidada, la obra permite hacerse una idea muy completa de ese periodo de transición. El debate arquitectónico se sitúa hoy en otras dimensiones, y no solo por el paso a lo digital, sino por el perfil de una profesión que se ha trasformado en las dos décadas que llevamos del siglo XX. La relación con la sociedad y los modos de producir arquitectura son otros.

La convocatoria de la presente edición de PpA versa sobre arquitecturas ampliadas y podría tener como referente inmediato la torre Bois-Le Prête (París) de 2011, obra de Lacaton \& Vassal, Premio Pritzker 2021. No obstante, los intereses del autor del ensayo en este caso entran de lleno en la convocatoria, pues incluyen el crecimiento urbano, las arquitecturas añadidas y, en general, las intervenciones sobre arquitecturas previas, de ahí que sea pertinente en el contexto del PpA 24. 

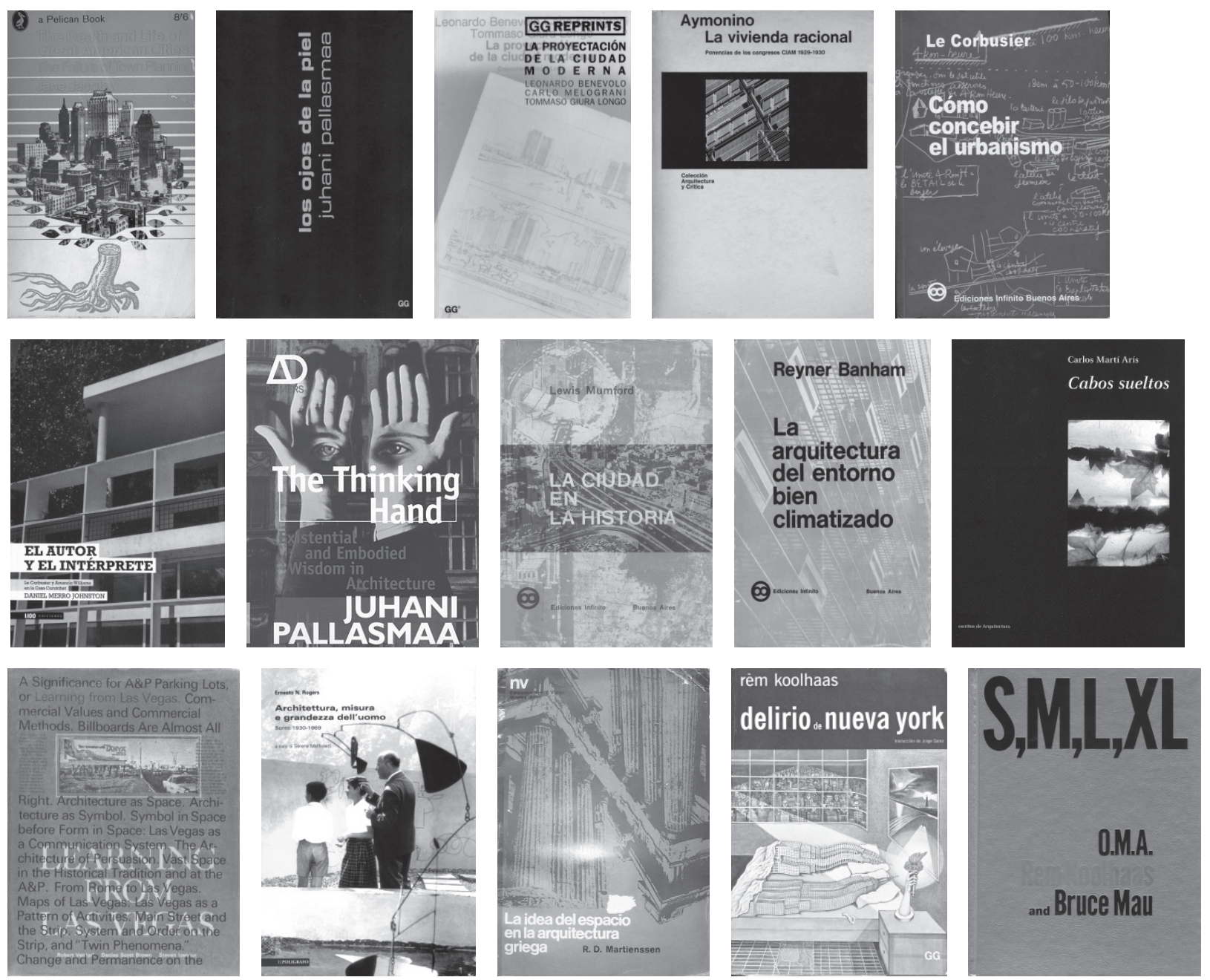

PPA N04: Jane Jacobs: MUERTE Y VIDA DE LAS GRANDES CIUDADES - Juhani Pallasmaa: LOS OJOS DE LA PIEL. LA ARQUITECTURA DE LOS SENTIDOS - Leonardo Benevolo et alt: LA PROYECTACIÓN DE LA CIUDAD MODERNA

PPA N05: Carlo Aymonino: LA VIVIENDA RACIONAL. PONENCIAS DE LOS CONGRESOS CIAM - Le Corbusier: CÓMO CONCEBIR EL URBANISMO - Daniel Merro Johnston: EL AUTOR Y EL INTÉRPRETE. LE CORBUSIER Y AMANCIO WILLIMAS EN LA CASA CURUTCHET

PPA N06: Juhani Pallasmaa: THE THINKING HAND: EXISTENTIAL AND EMBOIDIED WISDOM IN ARCHITECTURE - Lewis Mumford: LA CIUDAD EN LA HISTORIA. SUS ORÍGENES, TRANSFORMACIONES Y PERSPECTIVAS - Reyner Banham: LA ARQUITECTURA DEL ENTORNO BIEN CLIMATIZADO

PPA N07: Carlos Martí Arís: CABOS SUELTOS

PPA N08: Robert Venturi, Denise Scott Brown y Steven Izenour: LEARNING FROM LAS VEGAS - Serena Mafioletti: ARCHITTETURA, MISURA E GRANDEZA DELL'UOMO. SCRITTI 1930-1969

PPA N09: R. D. Martienssen: LA IDEA DEL ESPACIO EN LA ARQUITECTURA GRIEGA

PPA N10: Rem Koolhaas: SMALL, MEDIUM, LARGE, EXTRA-LARGE - Rem Koolhaas: DELIRIO DE NUEVA YORK. UN MANIFIESTO RETROACTIVO PARA MANHATTAN 

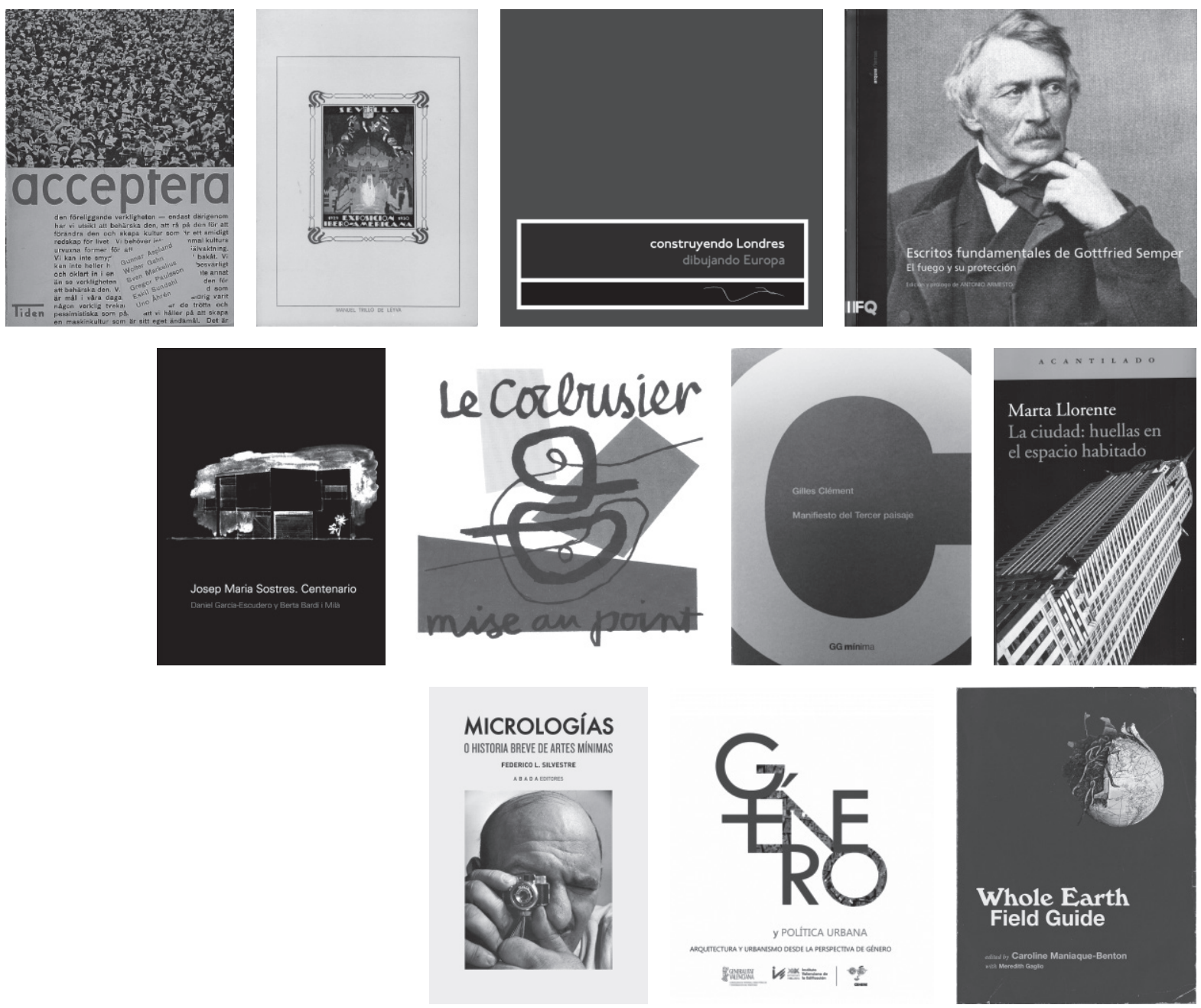

PPA N11: G. Asplund, W. Gahn, S. Markelius, G. Paulsson, E. Sundahl, U. Åhrén: ACCEPTERA

PPA N12: Manuel Trillo de Leyva: LA EXPOSICIÓN IBEROAMERICANA: LA TRANSFORMACIÓN URBANA DE SEVILLA - Manuel Trillo de Leyva: CONSTRUYENDO LONDRES; DIBUJANDO EUROPA

PPA N13: Antonio Armesto (Ed. y Prol.): ESCRITOS FUNDAMENTALES DE GOTTFRIED SEMPER. EL FUEGO Y SU PROTECCIÓN - Daniel García-Escudero y Berta Bardí i milà (Comps.): JOSÉ MARÍA SOSTRES. CENTENARIO - Jorge Torres Cueco (Trad.): LE CORBUSIER. MISE AU POINT

PPA N14: Gilles Clément: MANIFIESTO DEL TERCER PAISAJEERA - Marta Llorente Díaz: LA CIUDAD: HUELLAS EN EL ESPACIO HABITADO PPA N15: Federico López Silvestre: MICROLOGÍAS O BREVE HISTORIA DE ARTES MÍNIMAS

PPA N16: Begoña Serrano Lanzarote; Carolina Mateo Cecilia; Alberto Rubio Garrido (ED.): GÉNERO Y POLíTICA URBANA. ARQUITECTURA Y URBANISMODESDE LA PERSPECTIVA DE GÉNERO - Caroline Maniaque-Benton with Merodith Gaglio (EDS.) WHOLE EARTH FIELD GUIDE 

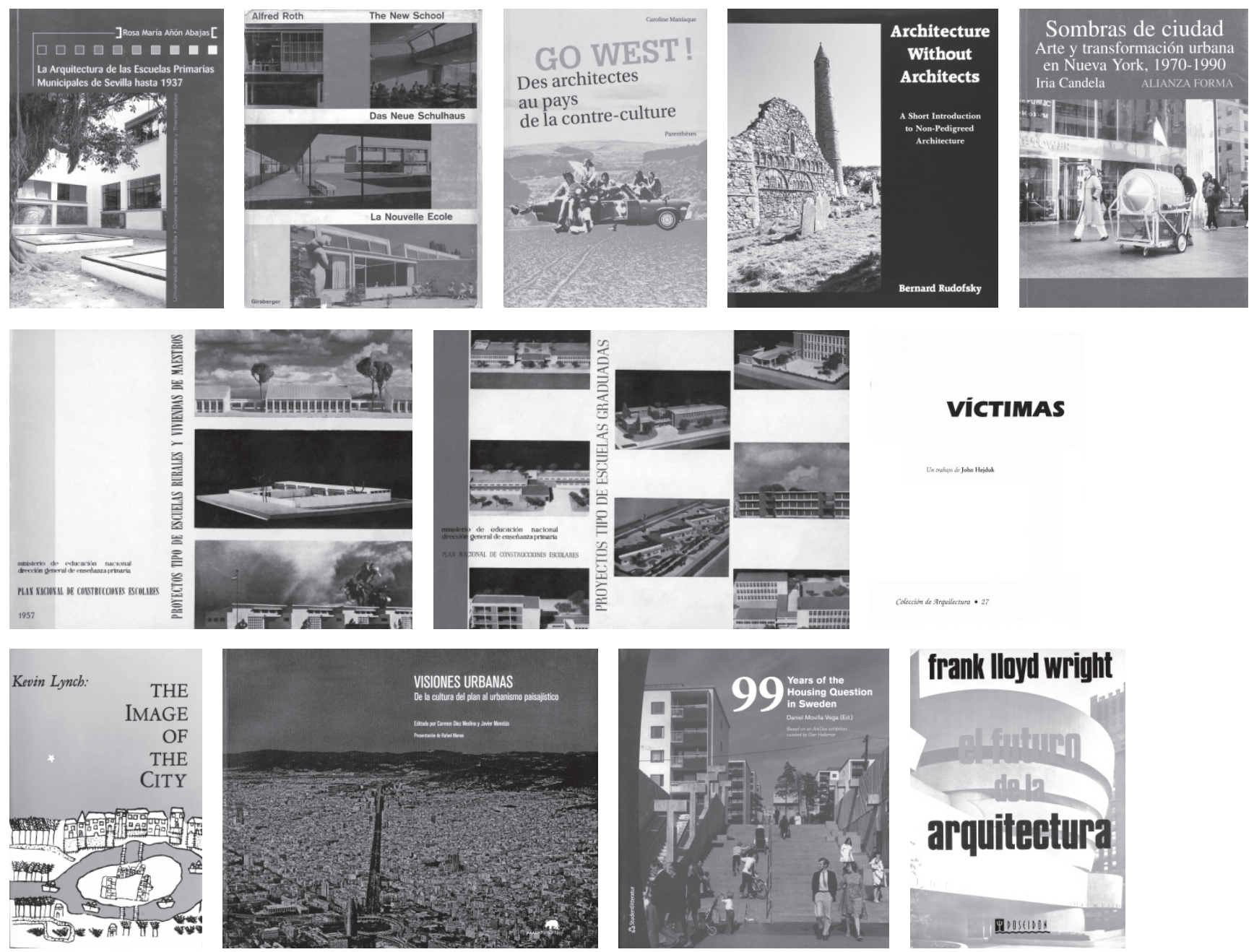

PPA N17: Rosa María Añón Abajas: LA ARQUITECTURA DE LAS ESCUELAS PRIMARIAS MUNICIPALES DE SEVILLA HASTA 1937 - Alfred Roth: THE NEW SCHOOL PLAN NACIONAL DE CONSTRUCCIONES ESCOLARES (VOLUMEN I) PROYECTOS TIPO DE ESCUELAS RURALES Y VIVIENDAS DE MAESTROS. PLAN NACIONAL DE CONSTRUCCIONES ESCOLARES (VOLUMNE II) PROYECTOS TIPO DE ESCUELAS GRADUADAS

PPA N18: Caroline Maniaque: GO WEST! DES ARCHITECTES AU PAYS DE LA CONTRE-CULTURE - Bernard Rudofsky: ARCHITECTURE WITHOUT ARCHITECTS. A SHORT INTRODUCTION TO NON-PEDIGREED ARCHITECTURE - Iria Candela: SOMBRES DE CIUDAD. ARTE Y TRANSFORMACIÓN URBANA EN NUEVA YORK 1970-1990

PPA N19: John Hejduk: VíCTIMAS - Kevin Lynch: THE IMAGE OF THE CITY - Carmen Díez Medina; Javier Monclús Fraga (eds): VISIONES URBANAS DE LA CULTURA DEL PLAN AL URBANISMO PAISAJÍSTICO

PPA N20: Daniel Movilla VEGA (Ed): 99 YEARS OF THE HOUSING QUESTION IN SWEDEN - Frank Lloyd Wright: EL FUTURO DE ARQUITECTURA 

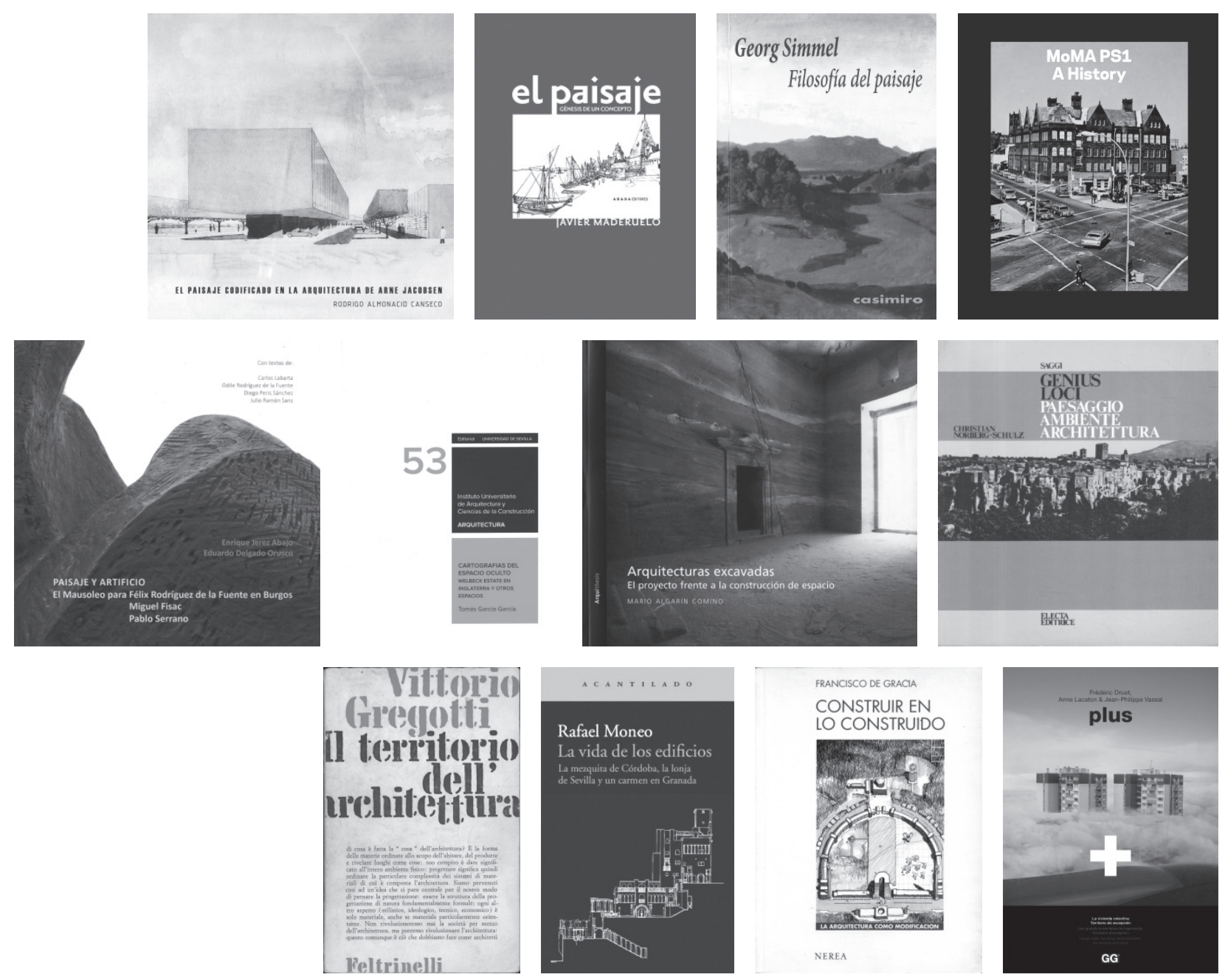

PPA N21: Rodrigo Almonacid Canseco: EL PAISAJE CODIFICADO EN LA ARQUITECTURA DE ARNE JACOBSEN - Javier Maderuelo: EL PAISAJE. GÉNESIS DE UN CONCEPTO - Georg Simmel: FILOSOFÍA DEL PAISAJE

PPA N22: Klaus Biesenbach y Betina Funcke (ed.): MOMA PS1. A HISTORY - Enrique Jerez Abajo y Eduardo Delgado Orusco: PAISAJE Y ARTIFICIO. EL MAUSOLEO PARA FÉLIX RODRÍGUEZ DE LA FUENTE EN BURGOS. MIGUEL FISAC, PABLO SERRANO - Tomás García García: CARTOGRAFÍAS DEL ESPACIO OCULTO. WELBECK ESTATE EN INGLATERRA Y OTROS ESPACIOS

PPA N23: Mario Algarín Comino: ARQUITECTURAS EXCAVADAS: EL PROYECTO FRENTE A LA CONSTRUCCIÓN DEL ESPACIO - Christian Norberg-Schulz: GENIUS LOCI: PAESAGGIO, AMBIENTE, ARCHITETTURA - Vittorio Gregotti: IL TERRITORIO DELL'ARCHITETTURA

PPA N24: Rafael Moneo Vallés: LA VIDA DE LOS EDIFICIOS. LA MEZQUITA DE CÓRDOBA, LA LONJA DE SEVILLA Y UN CARMEN EN GRANADA - Francisco de Gracia: CONSTRUIR EN LO CONSTRUIDO. LA ARQUITECTURA COMO MODIFICACIÓN - Frédéric Druot, Anne Lacaton y Jean Philippe Vassal: PLUS. LA VIVIENDA COLECTIVA. TERRITORIO DE EXCEPCIÓN. 

CITY. Darío Álvarez Álvarez • LINAZASORO EN REIMS. EL ESPACIO PÚBLICO COMO MEMORIA DEL LUGAR / LINAZASORO IN REIMS. THE PUBLIC SPACE AS THE MEMORY OF THE PLACE. Victoriano Sainz Gutiérrez • EDITAR VS. CONSTRUIR: UNA ECOLOGÍA DE LO INVISIBLE. AMPLIFICAR LA COMPRENSIÓN DE LAS TÉCNICAS DE PROYECTO / EDITING VS. BUILDING: AN ECOLOGY OF THE INVISIBLE. AMPLIFYING THE UNDERSTANDING OF ARCHITECTURAL DESIGN TECHNIQUES. Paula Victoria Álvarez Benítez • CUANDO LA PINTURA AMPLÍA LA ARQUITECTURA: INTERVENCIONES REALIZADAS EN EL ESPACIO PÚBLICO / WHEN PAINTING ENHANCES ARCHITECTURE: INTERVENTIONS IN A PUBLIC SETTING. Aurora Alcaide-Ramírez; Ana Ruiz-Abellón • UN EDIFICIO INVISIBLE. NUEVO AULARIO DE LA FACULTAD DE DERECHO DE LA UNIVERSIDAD DE ZARAGOZA (1983-1996) / AN INVISIBLE BUILDING. NEW LECTURE ROOM BUILDING OF THE FACULTY OF LAW OF THE UNIVERSITY OF ZARAGOZA (1983-1996). Luis Miguel Lus-Arana; Lucía Carmen Pérez-Moreno • ARQUITECTURAS AMPLIADAS. EL PABELLÓN DE EXPOSICIONES EN LA CASA DE CAMPO DE MADRID) / EXPANDED ARCHITECTURES. THE EXHIBITION PAVILION AT THE CASA DE CAMPO IN MADRID. José de Coca Leicher $\bullet$

- RAFAEL MONEO VALLÉS: LA VIDA DE LOS EDIFICIOS. LA MEZQUITA DE CÓRDOBA, LA LONJA DE SEVILLA Y UN CARMEN EN GRANADA . Víctor Pérez Escolano • FRANCISCO DE GRACIA: CONSTRUIR EN LO CONSTRUIDO. LA ARQUITECTURA COMO MODIFICACIÓN. Pablo Diañez Rubio • FRÉDÉRIC DRUOT, ANNE LACATON \& JEAN-PHILIPPE VASSAL PLUS: LA VIVIENDA COLECTIVA. TERRITORIO DE EXCEPCIÓN. Javier Terrados Cepeda.
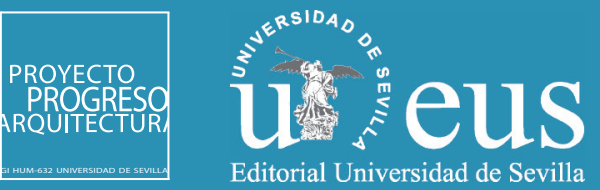